Methods Cases of cholangiocarcinoma were identified in Norwich (years 2004-2010) and Leicester (year 2007) from multi-disciplinary team meeting clinical databases. Inclusion required diagnostic evidence from CT scans and/or histology. Controls were patients, of similar ages and gender, with basal cell carcinomas treated in the dermatology departments at each hospital. The case notes of all subjects were reviewed to obtain confirmatory clinical information on cholangiocarcinoma and type 2 diabetes. Data were analysed using unconditional logistic regression to calculate ORs with $95 \%$ CIs, adjusted for age at diagnosis and gender.

Results A total of 80 cases of cholangiocarcinoma (median age at diagnosis $=76 \mathrm{yrs}$, range $41-96$ years, $51 \%$ men) and 411 controls were identified. All patients had radiological evidence of cancer, with $86 \%$ involving the extrahepatic biliary system. The median survival of cases was 158 days (range 2-1092 days). There was a statistically significant increase in the odds of developing cholangiocarcinoma for those with type 2 diabetes ( $O R=3.00,95 \%$ CI 1.44 to 6.25), but not for type $1(\mathrm{OR}=1.62,95 \% \mathrm{CI} 0.165$ to 16.08$)$. When the effect of type 2 diabetes was adjusted for use of oral hypoglycaemics, the associations were maintained (metformin $\mathrm{OR}=3.60,95 \%$ CI 1.26 to 10.25 and sulphonylureas, $\mathrm{OR}=6.31,95 \% \mathrm{CI} 2.31$ to 17.18 ).

Conclusion This epidemiological data supports the biological evidence for type 2 diabetes promoting the development of cholangiocarcinoma. Type 2 diabetes should be considered as a potential risk factor for cholangiocarcinoma in future aetiological studies.

Competing interests None declared.

\section{PTU-091 PERCUTANEOUS TRANSHEPATIC CHOLANGIOGRAPHY (PTC); ARE WE HITTING THE TARGET?}

doi:10.1136/gutjnl-2012-302514c.91

N J Taylor,* G Diana, M W James. Nottingham Digestive Diseases Biomedical Research Unit, Nottingham University Hospitals, Nottingham, UK

Introduction Percutaneous transhepatic cholangiography (PTC) is a procedure used to access the biliary tree for diagnostic purposes in obstructive jaundice, and to facilitate palliative stenting across biliary strictures. A previous study has shown a $19.8 \%$ in-patient mortality and significant morbidity for PTC. ${ }^{1}$ Our aim was to audit local practice in order to identify risk factors for complications and death and to identify ways of reducing patient exposure to PTC.

Methods Retrospective Audit of all patients presenting to Nottingham University Hospitals from 1 October 2010 to 31 December 2010. Patient demographics, indication for PTC, comorbidities, previous ERCP procedures performed, length of stay, PTC procedural details, morbidity and 30-day mortality was documented from electronic and paper records.

Results 44 patients underwent 72 procedures with a median of 1.5 procedures per patient. Median age was 67 years (IOR 62-77), 42\% were female and malignant disease was seen in $91 \%$ of patients undergoing PTC. In $68 \%$ of procedures the patients had one or more co-morbid conditions. $47 \%$ of procedures were for $1^{\text {st }}$ PTC and $53 \%$ were for 2nd or subsequent PTC. Overall, failed ERCP was the indication in $36 \%$ of procedures, reasons for ERCP failure were; failed cannulation $(50 \%)$; inability to cross stricture $(25 \%)$; hilar stricture (11\%). Median length of stay was 10 days for all procedures, two patients went home the day of procedure. Frequencies for each indication for PTC were; $38 \%$ distal; $27 \%$ mid-CBD/CHD stricture; $8 \%$ hilar stricture; $7 \%$ multiple strictures; $20 \%$ other. Intended drainage was achieved in all but one patient and the stricture was crossed in $93 \%$ procedures. Median pre-procedural bilirubin was $184 \mu \mathrm{mol} / 1$ (IOR 78-271) with a delta bilirubin at $72 \mathrm{~h}$ post PTC of $33 \mu \mathrm{mol} / \mathrm{l}(2-89)$. Overall complications were seen in $41 \%$ patients; $17 \%$ minor complications (pain and biliary sepsis) and $24 \%$ suffered major complications including severe sepsis and renal failure. 30 -day mortality was $18 \%$ with $13 \%$ being secondary to complications. Median survival overall was 182 days (IOR 81-321). Association with early death were Age*, $\geq 1$ co-morbidities*, $72 \mathrm{~h}$ post-procedural creatinine*, complications, hilar stricture $^{* *}$ and pre-procedural eGFR** $\left.{ }^{*} \mathrm{p}<0.05,{ }^{* *} \mathrm{p}<0.01\right)$.

Conclusion PTC is associated with a high incidence of complications and 30-day mortality. Risk factors for poor outcome include patient age; co-morbidity and renal function. Outcomes will likely be improved by better patient selection and pre-procedural optimisation. MRCP as part of the diagnostic pathway may identify strictures that should proceed directly to PTC and those where definitive stenting with cytology can be offered as a single-step procedure.

Competing interests None declared.

\section{REFERENCE}

1. Uberoi R, et al. British Society of Interventional Radiology: Biliary Drainage and Stenting Registry (BDSR). Cardiovasc Intervent Radiol 2011.

\section{Inflammatory bowel disease II PTU-092 CROHN'S DISEASE ASSOCIATED NOD2 VARIANTS SHOW DIFFERENTIAL ACTIVATION OF NF-KB IN RESPONSE TO AUTO-SIGNALLING AND MURAMYL DIPEPTIDE}

doi:10.1136/gutjnl-2012-302514c.92

\begin{abstract}
${ }^{1,2,3} \mathrm{~J}$ Van Limbergen, ${ }^{*} \mathrm{~F}$ Soares, ${ }^{5} \mathrm{R}$ K Russell, ${ }^{4} \mathrm{~S}$ Girardin, ${ }^{2} \mathrm{~A}$ Griffiths, ${ }^{3} \mathrm{D}$ Philpott. ${ }^{1}$ Child Life and Health, University of Edinburgh, Edinburgh, UK; ${ }^{2}$ Division of Gastroenterology, Hepatology and Nutrition, Toronto Sick Kids, Toronto, Canada; ${ }^{3}$ Department of Immunology, University of Toronto, Toronto, Canada; ${ }^{4}$ Laboratory Medicine and Pathobiology, University of Toronto, Toronto, Canada; ${ }^{5}$ Department of Paediatric Gastroenterology, Yorkhill Children's Hospital, Glasgow, UK
\end{abstract}

Introduction Single nucleotide polymorphisms (SNPs) located of the NOD2/CARD15 gene (nucleotide-binding oligomerization domain containing 2 /caspase recruitment domain family, member 15) are associated with increased susceptibility to Crohn's disease (CD). These SNPs are thought to disrupt the sensing of bacterial muramyl dipeptide (MDP) at the C-terminus of the NOD2 protein. The precise contribution of each of these SNPs (SNP5, 8, 12 and 13) to NF- $\kappa B$ activation by means of NOD2-auto-signalling and stimulation with MDP has not been investigated at low levels of NOD2 expression. Data regarding the linkage disequilibrium (LD) between these CD-associated SNPs are scarce.

Methods NOD2 variant constructs (rs2066842 (SNP5), rs2066844 (SNP8), rs2066845 (SNP12) and rs2066847 (SNP13), SNP5+8, SNP5 +12 and SNP5+13) were created by site-directed mutagenesis of a pCMV plasmid containing wild-type N-terminal FLAG-NOD2. NF$\kappa B$ luciferase assays were performed on HEK293 cells following transient transfection $(20 \mathrm{~h})$ with wildtype (WT) and NOD2 variant constructs, titrating NOD2 from 1 to $100 \mathrm{ng} /$ well. The NF$\kappa B$ luciferase response of NOD2 (1 ng)-transfected HEK293 cells to MDP $(10 \mu \mathrm{g} /$ well) was measured. Two-way ANOVA and unpaired ttests were used. By means of Haploview-analysis of sequencing data of the exons and exon-intron boundaries in 24 paediatric Caucasian Crohn's disease patients, we assessed the LD between SNP5 and SNP8, 12 and 13.

Results Two-way ANOVA demonstrated an effect of NOD2 genotype and concentration on auto-signalling at low levels of expression $(p<0.0001)$. This was due to the significant difference of autoactivation between WT and SNP5, SNP8 and SNP12 ( $<<0.001)$. At low levels of NOD2 expression (1-2 ng), the presence of SNP5 modified the auto-activating potential of SNP12 $(p<0.01)$. Based on these titration experiments, a low NOD2 transfection of $1 \mathrm{ng} /$ well was chosen for the MDP-stimulation experiment. MDP stimulation 